\title{
Time-dependent survival probability in diffusion-controlled reactions in a polymer chain: Beyond the Wilemski-Fixman theory
}

\author{
Goundla Srinivas \\ Solid State and Structural Chemistry Unit, Indian Institute of Science, Bangalore, India 560012 \\ K. L. Sebastian \\ Inorganic and Physical Chemistry Department, Indian Institute of Science, Bangalore, India 560012 \\ Biman Bagchia) \\ Solid State and Structural Chemistry Unit, Indian Institute of Science, Bangalore, India 560012
}

\begin{abstract}
Brownian dynamics simulation results of the time-dependent survival probability $\left(S_{p}(t)\right)$ of a donor-acceptor pair embedded at the two ends of a Rouse chain are compared with two different theories, one of which is the well-known Wilemski-Fixman (WF) theory. The reaction studied is fluorescence energy transfer via the Förster mechanism, which has a $R^{-6}$ distance $(R)$ dependence of the reaction rate. It has been reported earlier [G. Srinivas, A. Yethiraj, and B. Bagchi, J. Chem. Phys. 114, 9170 (2001)] that while the WF theory is satisfactory for small reaction rates, the agreement was found to become progressively poorer as the rate is increased. In this work, we have generalized the WF theory. We suggest an approximate, reduced propagator technique for three-dimensional treatment (instead of $3 N$ dimensions, where $N$ is the number of monomers in the polymer chain). This equation is solved by combining a Green's function solution with a discretized sink method. The results obtained by this new scheme are in better agreement with the simulation results.
\end{abstract}

\section{INTRODUCTION}

Reactions between any two sites of a polymer chain have been a subject of great interest. ${ }^{1-8}$ Often these reactions occur via the distance dependent rate, such as fluorescence resonance energy transfer (FRET) by the long distance Förster mechanism, ${ }^{1,9-14}$ electron transfer reactions, ${ }^{15,16}$ etc. The mechanism of long distance FRET is Coulombic and is usually discussed in terms of Förster theory ${ }^{1,2}$ which gives the following distance dependent rate of energy transfer,

$$
k_{f}(R)=k_{\mathrm{rad}}\left(\frac{R_{\mathrm{F}}}{R}\right)^{6},
$$

where $R_{\mathrm{F}}$ is the Förster radius, defined as the D-A separation corresponding to $50 \%$ energy transfer. $k_{\text {rad }}$ is the radiative rate, which is typically of the order of $10^{8}$ to $10^{9} \mathrm{~cm}^{-1}$ for the commonly used chromophores in FRET experiments. According to the above equation $k_{\text {rad }}$ can be understood as the rate of energy transfer when the separation between the donor and the acceptor is equal to Förster radius (i.e., $R / R_{\mathrm{F}}$ $=1$ ). The Förster radius is usually obtained from the overlap of the donor fluorescence with the acceptor absorption and several other available parameters. ${ }^{9,10}$

The dynamics of Förster energy migration has been investigated traditionally via time domain measurements of the decay of the fluorescence (due to excitation transfer) from the donor. ${ }^{1,10}$ As both $k_{\text {rad }}$ and $R_{\mathrm{F}}$ are determined by the D-A pair, the rate of decay of the fluorescence intensity provides a direct probe of the conformational dynamics of the polymer.

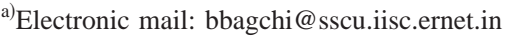

Recently, this technique has been used in single molecule spectroscopy ${ }^{17}$ of biopolymers ${ }^{11-14}$ and proteins, ${ }^{11}$ where the distance dependence of FRET provides relevant information about the conformation and dynamics of single biopolymers. At any given time after the initial excitation, the fluorescence intensity is a measure of the "unreacted" donor concentration, that is, of the survival probability, $S_{p}(t)$.

The complexity of describing the dynamics of energy transfer of polymers in solution arises from the fact that, due to chain connectivity, the Brownian motion of the monomers on the polymer are strongly correlated. The many-body nature of polymer dynamics can be described by a joint, timedependent probability distribution $P\left(\mathbf{r}^{N}, t\right)$ where $\mathbf{r}^{N}$ denotes the position of all the $N$ polymer beads, at time $t$. The time dependence of the probability distribution $P\left(\mathbf{r}^{N}, t\right)$ can be described by the following reaction-diffusion equation, ${ }^{4,5}$

$$
\frac{\partial}{\partial t} P\left(\mathbf{r}^{N}, t\right)=\mathcal{L}_{B}\left(\mathbf{r}^{N}\right) P\left(\mathbf{r}^{N}, t\right)-k(R) P\left(\mathbf{r}^{N}, t\right),
$$

where $\mathcal{L}_{B}$ is the full $3 N$ dimensional diffusion operator,

$$
\mathcal{L}_{B}\left(\mathbf{r}^{N}\right) P\left(\mathbf{r}^{N}, t\right)=D \sum_{j=1}^{N} \frac{\partial}{\partial r_{j}} P_{\mathrm{eq}}\left(\mathbf{r}^{N}\right) \frac{\partial}{\partial r_{j}} \frac{1}{P_{\mathrm{eq}}\left(\mathbf{r}^{N}\right)} P\left(\mathbf{r}^{N}, t\right)
$$

the subscript "eq" denotes equilibrium, $R$ is the scalar distance between the two ends of the polymer chain, and $D$ is the diffusion coefficient of a monomer The solution of Eq. (2), with the sink term [last term on the right-hand side of Eq. (2)] given by the Förster expression ${ }^{1,2}$ for $k(R)$ is highly nontrivial. 
In two seminal papers, Wilemski and Fixman (WF) ${ }^{4,5}$ presented a nearly analytic solution of the problem for any arbitrary sink. Pastor, Zwanzig, and Szabo ${ }^{3,18}$ tested the WF theory only for the average rate, by computer simulations when the sink is a Heaviside function. They found that the WF theory is efficient for the sinks with smaller radii. In spite of its importance, the WF theory has never been studied for a distance dependence rate, such as Förster energy transfer. Such a study is clearly important because the end-to-end probability distribution in polymer, peaks at a distance which scales as $N^{2 \nu} . \nu=1 / 2$ for the Rouse chain and $3 / 5$ for the self-avoiding walk (SAW). ${ }^{19}$

Recently, we have carried out a Brownian dynamics simulation study of the dynamics of energy transfer in Rouse chain. ${ }^{8}$ The polymer molecule was modeled as an ideal Gaussian chain with $N$ monomer units with segment (or Kuhn) length $b$. The donor and acceptor sites were assumed to be located at opposite ends of the polymer chain. The resonance energy transfer rate, $k(R)$ was assumed to be given by,

$$
k(R)=\frac{k_{\mathrm{rad}}}{1+\left(R / R_{\mathrm{F}}\right)^{6}} .
$$

Note that the above-mentioned form is different from the commonly used form of the Forster rate, $k_{f}(R)$ given by Eq. (1). The $\left(R_{\mathrm{F}} / R\right)^{6}$ distance dependence is not appropriate here, since it diverges at $R \rightarrow 0$, which is allowed in Rouse chain, ${ }^{20,21}$ but not in a real polymer, where the end-to-end distance $(R)$ never approaches zero, due to the excluded volume forces. Thus, the modified form [Eq. (4)] used is reasonable. The reason that we use Rouse chain is that this case can be treated easily in theory. For example, the theory of Wilemski and Fixman ${ }^{4,5,22}$ can be readily applied to the Rouse chain, because the necessary Green's function is available in analytic form.

It was reported in Ref. 8 that the Wilemski-Fixman theory, unfortunately, does not provide a satisfactory description for the following situations: (a) When the rate $k_{\text {rad }}$ is much larger than the rate of monomer diffusion, given by $b^{2} / D$; (b) when $R_{\mathrm{F}}$ is close to the separation where the probability of finding the chain ends is maximum. The above limitations of the WF theory were somewhat surprising and motivated the present work.

The main objectives of this paper are the following: (1) to present further Brownian dynamics (BD) simulations of Eq. (2), with $k(R)$ given by the Förster rate [Eq. (4)]; (2) to present an alternative theoretical approach to treat the dynamics of FRET in polymers.

The new theoretical approach employs a reduced, threedimensional equation-of-motion. The solution of the threedimensional equation-of-motion employs a novel reduction of the equation-of-motion to one-dimension and subsequently uses a discretized sink model to obtain the survival probability. Henceforth, this method will be referred to as the three-dimensional reduced Green's function method (RGF).

It is found that the agreement of the results obtained from the new scheme with the simulations is superior to that of Wilemski-Fixman. However, the agreement is still not perfect in several limiting cases. The reason for this has been discussed.

Simulation details and the description of the WilemskiFixman theory remain same as that in our previous study. ${ }^{8}$ The organization of the rest of the paper is as follows. In the next section, we introduce the RGF method. The implementation of this method through the discretized sink method is also presented in the same section. In Sec. III, the simulations results are compared with the theoretical predictions. Conclusions are presented in Sec. IV.

\section{THEORY}

\section{A. An approximate equation for the reduced Green's function}

The formal solution of Eq. (2) is

$$
P\left(\mathbf{r}^{N}, t\right)=\int d \mathbf{r}_{0}^{N} \mathrm{G}\left(\mathbf{r}^{N}, t \mid \mathbf{r}_{0}^{N}, 0\right) P\left(\mathbf{r}_{0}^{N}, 0\right) .
$$

$P\left(\mathbf{r}_{0}^{N}, 0\right)$ denotes the initial distribution and $\mathrm{G}\left(\mathbf{r}^{N}, t \mid \mathbf{r}_{0}^{N}, 0\right)$ is the Green's function for the problem. This is exact, but not very usable as it involves all the $\mathbf{r}^{N}$. One would like to have an equation involving only the relevant coordinate, $R$. We derive such an equation in the following. The derivation involves two steps. First, we derive an approximate equation involving only the end-to-end vector $\mathbf{R}$. Then, using the fact that the sink function depends only on the magnitude of $\mathbf{R}$, this is reduced exactly to a one-dimensional diffusion problem.

As we are interested only in the dynamics of $\mathbf{R}$, we refer to the remaining coordinates of the chain as the irrelevant coordinates and denote them by $\mathbf{S}$. Instead of using the variables $\mathbf{r}^{N}$, one can use $(\mathbf{R}, \mathbf{S})$. We now write $G\left(\mathbf{r}^{N}, t \mid \mathbf{r}_{0}^{N}, 0\right)$, in terms of $(\mathbf{R}, \mathbf{S})$, as $\mathcal{G}\left(\mathbf{R}, \mathbf{S}, t \mid \mathbf{R}_{0}, \mathbf{S}_{0}, 0\right)$. The differential Eq. (2), is equivalent to the exact integral equation:

$$
\begin{aligned}
\mathrm{G}\left(\mathbf{R}, \mathbf{S}, t \mid \mathbf{R}_{0}, \mathbf{S}_{0}\right) & \\
= & \mathrm{G}_{0}\left(\mathbf{R}, \mathbf{S}, t \mid \mathbf{R}_{0}, \mathbf{S}_{0}, 0\right)-\int_{0}^{t} d t_{1} \int d \mathbf{R}_{1} \int d \mathbf{S}_{1} \\
& \times \mathrm{G}_{0}\left(\mathbf{R}, \mathbf{S}, t \mid \mathbf{R}_{1}, \mathbf{S}_{1}, t_{1}\right) k\left(R_{1}\right) \mathrm{G}\left(\mathbf{R}_{1}, \mathbf{S}_{1}, t_{1} \mid \mathbf{R}_{0}, \mathbf{S}_{0}, 0\right) .
\end{aligned}
$$

$\mathrm{G}_{0}\left(\mathbf{R}, \mathbf{S}, t \mid \mathbf{R}_{0}, \mathbf{S}_{0}, 0\right)$ would be the Green's function if the sink is not present. We now introduce the conditional probability distribution $P_{\text {eq }}(\mathbf{S} \mid \mathbf{R})=P_{\text {eq }}(\mathbf{R}, \mathbf{S}) / P_{\text {eq }}(\mathbf{R})$, where we define the reduced equilibrium probability distribution for $\mathbf{R}$ by $P_{\text {eq }}(\mathbf{R})=\int d \mathbf{S} P_{\text {eq }}(\mathbf{R}, \mathbf{S})$. Multiplying Eq. (6) by $P_{\text {eq }}\left(\mathbf{S}_{0} \mid \mathbf{R}_{0}\right) d \mathbf{S}_{0} d \mathbf{S}$, and integrating over all initial and final positions of the irrelevant coordinates $\mathbf{S}_{0}$ and $\mathbf{S}$, we get

$$
\begin{aligned}
\mathrm{G}(\mathbf{R}, t \mid & \left.\mathbf{R}_{0}, 0\right) \\
= & \mathrm{G}_{0}\left(\mathbf{R}, t \mid \mathbf{R}_{0}, 0\right)-\int d \mathbf{S} \int_{0}^{t} d t_{1} \int d \mathbf{R}_{1} \int d \mathbf{S}_{1} \\
& \times \mathrm{G}_{0}\left(\mathbf{R}, \mathbf{S}, t \mid \mathbf{R}_{1}, \mathbf{S}_{1}, t_{1}\right) k\left(R_{1}\right) \int d \mathbf{S}_{0} \\
& \times \mathrm{G}\left(\mathbf{R}_{1}, \mathbf{S}_{1}, t_{1} \mid \mathbf{R}_{0}, \mathbf{S}_{0}, 0\right) P_{\mathrm{eq}}\left(\mathbf{S}_{0} \mid \mathbf{R}_{0}\right) .
\end{aligned}
$$


In the Eq. (7), we have defined the reduced Green's function for the $\mathbf{R}$ coordinate by

$$
\begin{aligned}
\mathrm{G}\left(\mathbf{R}, t \mid \mathbf{R}_{0}, 0\right)= & \int d \mathbf{S} \int d \mathbf{S}_{0} \mathrm{G}\left(\mathbf{R}, \mathbf{S}, t \mid \mathbf{R}_{0}, \mathbf{S}_{0}, 0\right) \\
& \times P_{\text {eq }}\left(\mathbf{S}_{0} \mid \mathbf{R}_{0}\right),
\end{aligned}
$$

with a similar definition for $\mathrm{G}_{0}\left(\mathbf{R}, t \mid \mathbf{R}_{0}, 0\right)$. Equation (7) cannot be solved. So we introduce the approximation

$$
\begin{aligned}
\int d \mathbf{S}_{0} \mathrm{G}\left(\mathbf{R}_{1}, \mathbf{S}_{1}, t_{1} \mid \mathbf{R}_{0}, \mathbf{S}_{0}, 0\right) P_{\text {eq }}\left(\mathbf{S}_{0} \mid \mathbf{R}_{0}\right) \\
\simeq P_{\text {eq }}\left(\mathbf{S}_{1} \mid \mathbf{R}_{1}\right) \int d \mathbf{S}_{1}^{\prime} \int d \mathbf{S}_{0} \mathrm{G}\left(\mathbf{R}_{1}, \mathbf{S}_{1}^{\prime}, t_{1} \mid \mathbf{R}_{0}, \mathbf{S}_{0}, 0\right) \\
\quad \times P_{\text {eq }}\left(\mathbf{S}_{0} \mid \mathbf{R}_{0}\right) .
\end{aligned}
$$

With this, Eq. (7) becomes

$$
\begin{aligned}
\mathrm{G}\left(\mathbf{R}, t \mid \mathbf{R}_{0}, 0\right)= & \mathrm{G}_{0}\left(\mathbf{R}, t \mid \mathbf{R}_{0}, 0\right)-\int_{0}^{t} d t_{1} \int d \mathbf{R}_{1} \\
& \times \mathrm{G}_{0}\left(\mathbf{R}, t \mid \mathbf{R}_{1}, t_{1}\right) k\left(R_{1}\right) \mathrm{G}\left(\mathbf{R}_{1}, t_{1} \mid \mathbf{R}_{0}, 0\right) .
\end{aligned}
$$

The approximation of Eq. (8) has the property that it is exact in the limits $t_{1} \rightarrow 0$ or $\infty$. The physical meaning of the approximation is that if, from the equilibrium ensemble, one selects only those that have an end-to-end vector equal to $\mathbf{R}_{0}$ and evolves them in time in presence of the sink, then the resultant probability for finding the system at $\mathbf{R}_{1}, \mathbf{S}_{1}$ may be approximated by the product of two terms. They are (i) the exact probability that the end to vector has a new value $\mathbf{R}_{1}$, and (ii) the conditional probability for finding the irrelevant coordinates at $\mathbf{S}_{1}$ given that the end-to-end vector has the value $\mathbf{R}_{1}$, calculated at equilibrium. This approximation would follow if one assumed that the irrelevant variables adjust to the instantaneous value of $\mathbf{R}$. One expects that approximation is reasonable for longer times, but would show deviations for shorter.

\section{B. Reduction to a one-dimensional equation}

The main idea used in the subsequent steps is that the Förster reaction rate depends only on the distance $R$ between the donor and the acceptor. Thus, the above threedimensional Eq. (9) can be reduced to a one-dimensional equation. This further reduction, however, requires some algebraic manipulations described below.

In Laplace frequency plane Eq. (9) takes the form,

$$
\begin{aligned}
\mathrm{G}\left(\mathbf{R}, z \mid \mathbf{R}_{0}\right)= & \mathrm{G}_{0}\left(\mathbf{R}, z \mid \mathbf{R}_{0}\right)-\int d \mathbf{R}^{\prime} \mathrm{G}_{0}\left(\mathbf{R}, z \mid \mathbf{R}^{\prime}\right) \\
& \times k\left(R^{\prime}\right) \mathrm{G}\left(\mathbf{R}^{\prime}, z \mid \mathbf{R}_{0}\right) .
\end{aligned}
$$

By multiplying the above equation by $d \boldsymbol{\Omega}_{\mathbf{R}}$ and integrating over all the orientations of $\mathbf{R}$ we get,

$$
\begin{aligned}
\mathcal{G}\left(R, z \mid R_{0}\right)= & \mathcal{G}_{1},\left(R, z \mid R_{0}\right)-\int_{0}^{\infty}\left(R^{\prime}\right)^{2} d R^{\prime} \mathcal{G}\left(R, z \mid R^{\prime}\right) \\
& \times k\left(R^{\prime}\right) \mathcal{G}\left(R^{\prime}, z \mid R_{0}\right),
\end{aligned}
$$

where we have defined two auxiliary functions:

$$
\mathcal{G}_{0}\left(R, z \mid R_{0}\right)=\int d \Omega_{\mathbf{R}} \mathrm{G}_{0}\left(\mathbf{R}, z \mid \mathbf{R}_{0}\right)
$$

and

$$
\mathcal{G}\left(R, z \mid R_{0}\right)=\int d \Omega_{\mathbf{R}} \mathrm{G}\left(\mathbf{R}, z \mid \mathbf{R}_{0}\right) .
$$

Our interest is in the survival probability,

$$
S_{p}(t)=\int d \mathbf{R} \int d \mathbf{R}_{0} \mathrm{G}\left(\mathbf{R}, t \mid \mathbf{R}_{0}\right) P_{\mathrm{eq}}\left(\mathbf{R}_{0}\right),
$$

using the fact that $P_{\text {eq }}\left(\mathbf{R}_{0}\right)$ depends only on $R_{0}$, we can write this as,

$$
S_{p}(t)=4 \pi \int_{0}^{\infty} R^{2} d R \int_{0}^{\infty} R_{0}^{2} d R_{0} \mathcal{G}\left(R, t \mid R_{0}\right) P_{\mathrm{eq}}\left(R_{0}\right) .
$$

Now it is convenient to define,

$$
\hat{\mathcal{P}}(R, z)=\int_{0}^{\infty} R_{0}^{2} d R_{0} \mathcal{G}\left(R, z \mid R_{0}\right) P_{\mathrm{eq}}\left(R_{0}\right) .
$$

Then, $\hat{\mathcal{P}}(R, z)$ obeys the equation

$$
\begin{aligned}
\hat{\mathcal{P}}(R, z)= & \frac{1}{z} \mathcal{P}_{\mathrm{eq}}(R)-\int_{0}^{\infty}\left(R^{\prime}\right)^{2} d R^{\prime} \mathcal{G}\left(R, z \mid R^{\prime}\right) \\
& \times k\left(R^{\prime}\right) \hat{\mathcal{P}}\left(R^{\prime}, z\right) .
\end{aligned}
$$

Equation (17) is now solved by employing a discretized sink method, developed earlier. ${ }^{23-26}$

\section{Discretized sink method}

In this method, the continuous sink curve is discretized into $M$ number of intervals. Thus, in discretized sink notation the sink function [defined by Eq. (4)] can be written as

$$
k(R)=k_{\mathrm{rad}} \sum_{s} \delta\left(R-R_{s}\right)\left(\frac{R_{\mathrm{F}}^{6}}{R_{s}^{6}+R_{\mathrm{F}}^{6}}\right) .
$$

Note that the summation is over all the sink points $(s)$, where the populations are given by $P\left(R_{s}, z\right)$. Equation (17) can be discretized as

$$
\begin{aligned}
\hat{\mathcal{P}}(R, z)= & \frac{1}{z} \mathcal{P}_{\mathrm{eq}}(R, z)-\sum_{s} R_{s}^{2} \mathcal{G}_{0}\left(R, z \mid R_{s}\right) \\
& \times k\left(R_{s}\right) \hat{\mathcal{P}}\left(R_{s}, z\right) .
\end{aligned}
$$

By finding $P\left(R_{s}, z\right)$ using this, a set of linear equations are generated. These can be written in matrix form as,

$$
\mathbf{B} \cdot \mathbf{P}=\mathbf{P}_{0},
$$

where the elements of matrices $\mathbf{B}, \mathbf{P}$, and $\mathbf{P}_{0}$ are given by,

$$
\begin{aligned}
& B_{m n}=\delta_{m n}+k_{n} R_{n}^{2} \mathcal{G}_{0}\left(R_{m}, z \mid R_{n}\right), \\
& P_{m}=P\left(R_{m}, z\right),
\end{aligned}
$$

and

$$
P_{0 m}=P_{0}\left(R_{m}, z\right),
$$

and $k_{n}=k\left(R_{n}\right)$. 


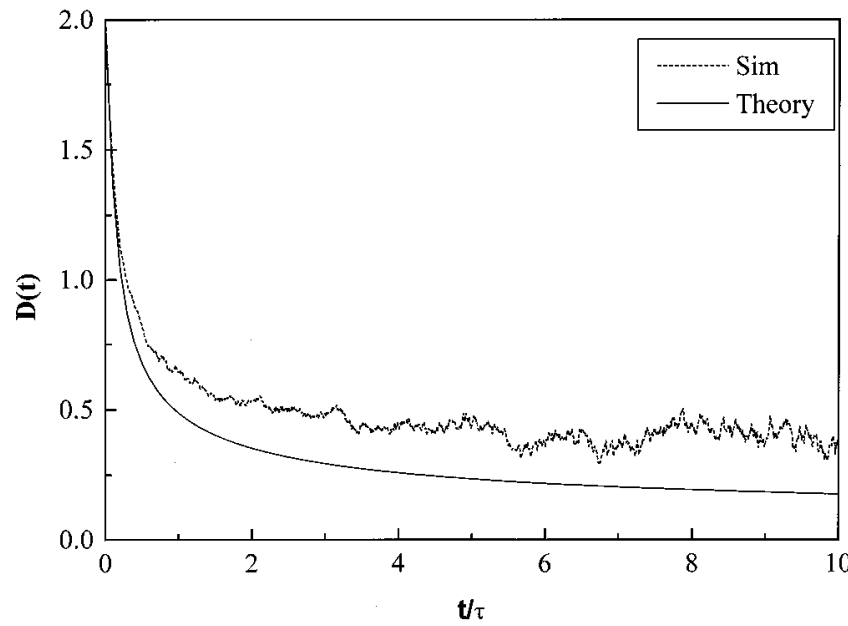

FIG. 1. The time-dependent diffusion of end-to-end separation obtained by using the $\rho(t)$ from Brownian dynamics simulations (dashed line) and the theory (full line; obtained by using the analytical $\rho(t)$ ) is plotted as a function of reduced time for $N=50$.

Now note that $\mathcal{G}\left(R, t \mid R_{0}\right)$, after averaging over the angles and using the Green's function definition (given in Appendix A), can be written in the following form

$$
\begin{aligned}
\mathcal{G}_{0}\left(R, t \mid R_{0}\right)= & \frac{1}{L R R_{0} \rho(t)} \sqrt{\frac{6}{\pi\left(1-\rho(t)^{2}\right)}} \\
& \times \sinh \left(\frac{3 R R_{0} \rho(t)}{L^{2}\left(1-\rho(t)^{2}\right)}\right) \\
& \times e^{-3\left(R^{2}+R_{0}{ }^{2} \rho(t)^{2}\right) / 2 L^{2}\left(1-\rho(t)^{2}\right)} .
\end{aligned}
$$

By taking the Laplace transform of $\mathcal{G}_{0}\left(R, t \mid R_{0}\right)$ and substituting in Eq. (19), we obtain $\hat{\mathcal{P}}(R, z)$. The resulting $\hat{\mathcal{P}}(R, z)$ can be used in the following equation to obtain the survival probability in $z$-plane.

$$
S_{p}(z)=\frac{1}{z}\left[1-\sum_{s} k\left(R_{s}\right) R_{s}^{2} \hat{\mathcal{P}}\left(R_{s}, z\right)\right] .
$$

Finally, the time-dependent survival probability can be obtained through the Laplace inversion,

$$
S_{p}(t)=\mathcal{L}^{-1}\left(S_{p}(z)\right) \text {. }
$$

\section{NUMERICAL RESULTS}

The discretized sink method can be conveniently used to solve Eq. (2) for a wide range of $k_{\mathrm{rad}}$ and $R_{\mathrm{F}}$ values. The method is fairly simple to implement and is not numerically intensive. In this section we present the results obtained by using RGF and compare with WF theory prediction, as well as with the BD simulation results.

In the reduced three-dimensional description the mutual diffusion becomes time-dependent. This can be expressed in terms of the end-to-end vector correlations function as follows,

$$
D(t)=-\frac{L^{2}}{3}\left(\frac{\partial}{\partial t} \ln \rho(t)\right) .
$$

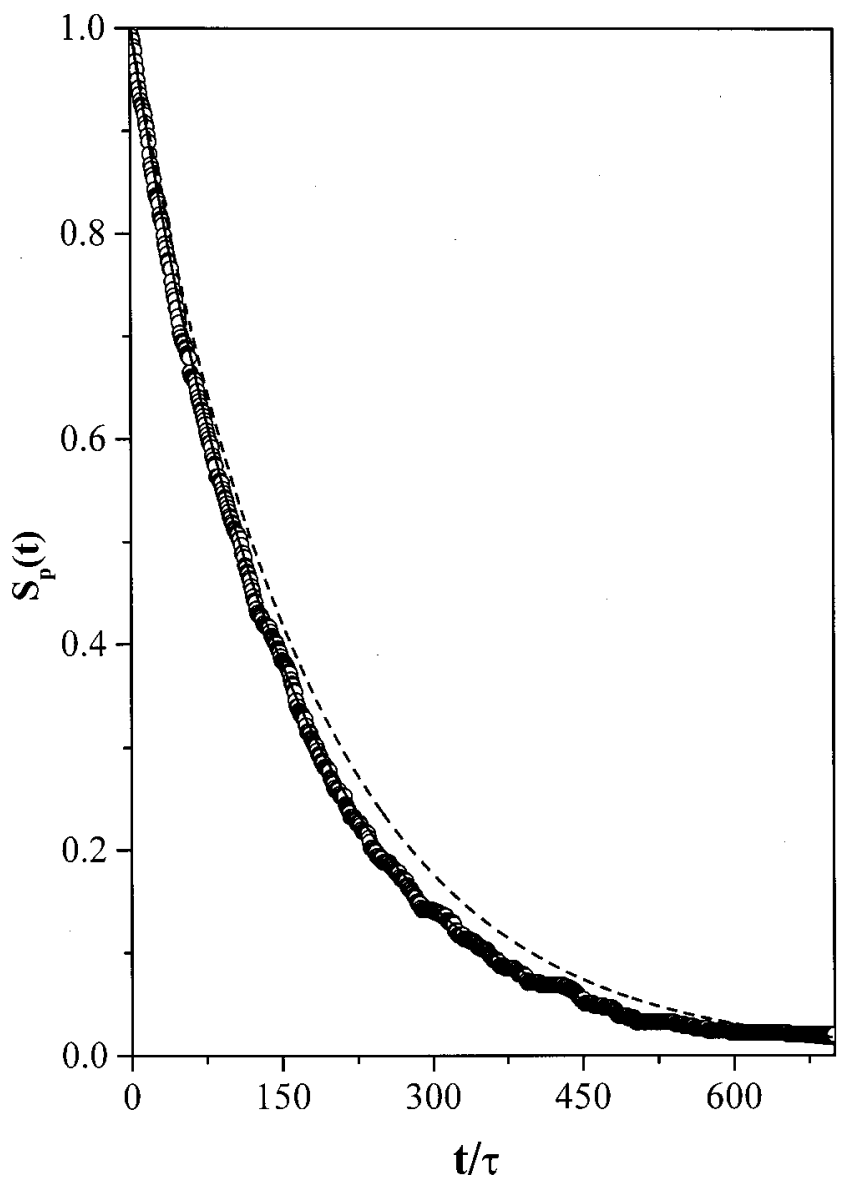

FIG. 2. Results of $S_{p}(t)$ are plotted against the reduced time for $R_{F}=1$ and $k_{\text {rad }}=1$ for $N=50$. The results obtained by simulation are shown with symbol, while the theoretical predictions are shown by line. The reduced Green's function method (RGF) (full line) results are in better agreement with simulation result over the prediction of the WF theory (dashed line).

Although $D(t)$ has not been directly used in the present study, it provides a useful measure of the end-to-end diffusion. In Fig. 1, we show the time dependence of the end-toend diffusion as a function of reduced time for $N=50$. It decays monotonically with time from its initial value. The full line corresponds to the theoretical result [obtained by using analytical $\rho(t)$; see Appendix B]. The dashed line is obtained by using simulated $\rho(t)$. As shown in the figure, the initial value, which correspond to the sum of the bare diffusion of the two ends $\left(2 D_{0}\right)$, decreases with time and saturates to a finite nonzero value in the long time limit (see Appendix B). Asymptotically, $D^{\text {sim }}(t)$ and $D^{\text {theory }}(t)$ approach somewhat different values in the $t \rightarrow \infty$ limit. This may be due to the finite size effects.

Figures 2 and 3 show the comparison between the survival probability obtained from the Brownian dynamics (BD) simulations and the theoretical prediction [Eq. (25)], for $k_{\mathrm{rad}}=1$. In both the figures, BD simulation results are shown by symbol, while the theoretical results are shown by line. The RGF and WF theory predictions are shown by full and dashed line, respectively. In Fig. 2 the survival probability for $R_{\mathrm{F}}=1$ is plotted against the reduced time. The representation of Fig. 3 is the same as that of Fig. 2, except that this figure is plotted for a larger $R_{\mathrm{F}}$ value, namely $R_{\mathrm{F}}=5$. 


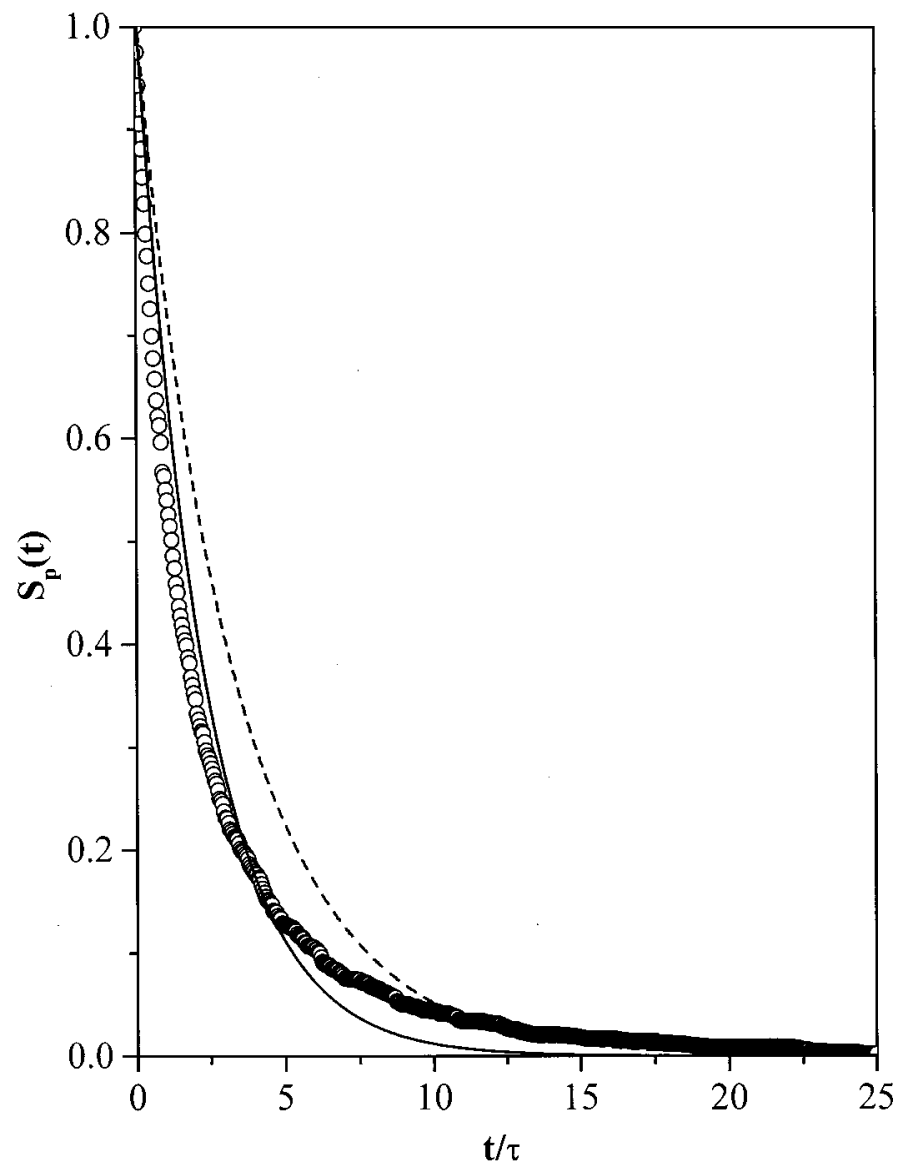

FIG. 3. Similar to Fig. 2 but for $R_{F}=5$. Though the agreement becomes poorer as $R_{F}$ is increased, RGF results (full line) are in better agreement with the simulation result (symbols) compared to WF theory prediction (dashed line)

Note that similar to the WF theory, the RGF results agree well with that of the simulation at smaller $R_{\mathrm{F}}$ values. However, the agreement with WF becomes progressively poorer with increasing $R_{\mathrm{F}}$. On the other hand, the RGF results are in better agreement with that of the simulations, even at large $R_{\mathrm{F}}$ values (shown in Fig. 3), where WF theory completely breaks down in the long times.

In Fig. 4 the survival probability for a larger radiative rate $\left(k_{\mathrm{rad}}=10\right)$ is plotted at $R_{\mathrm{F}}=1$. The RGF results (shown by full line) are again in better agreement with the simulation results (shown by symbols) than the WF theory (dashed line). However, the agreement with the present RGF method is not perfect.

The precise reason for the failure of our RGF is not clear at present. However, it is important to note that the reduced three-dimensional equation used in this study is an approximation. This is probably the main source of error in our treatment.

\section{CONCLUSIONS}

In view of the limitation of WF theory at large rates $^{8}$ we have generalized the WF theory by reducing the $3 N$ dimensional problem to three-dimensions. The resulting reduced Green's function equation is solved by using the discretized sink method to obtain the $S_{p}(t)$. Although the deficiency still

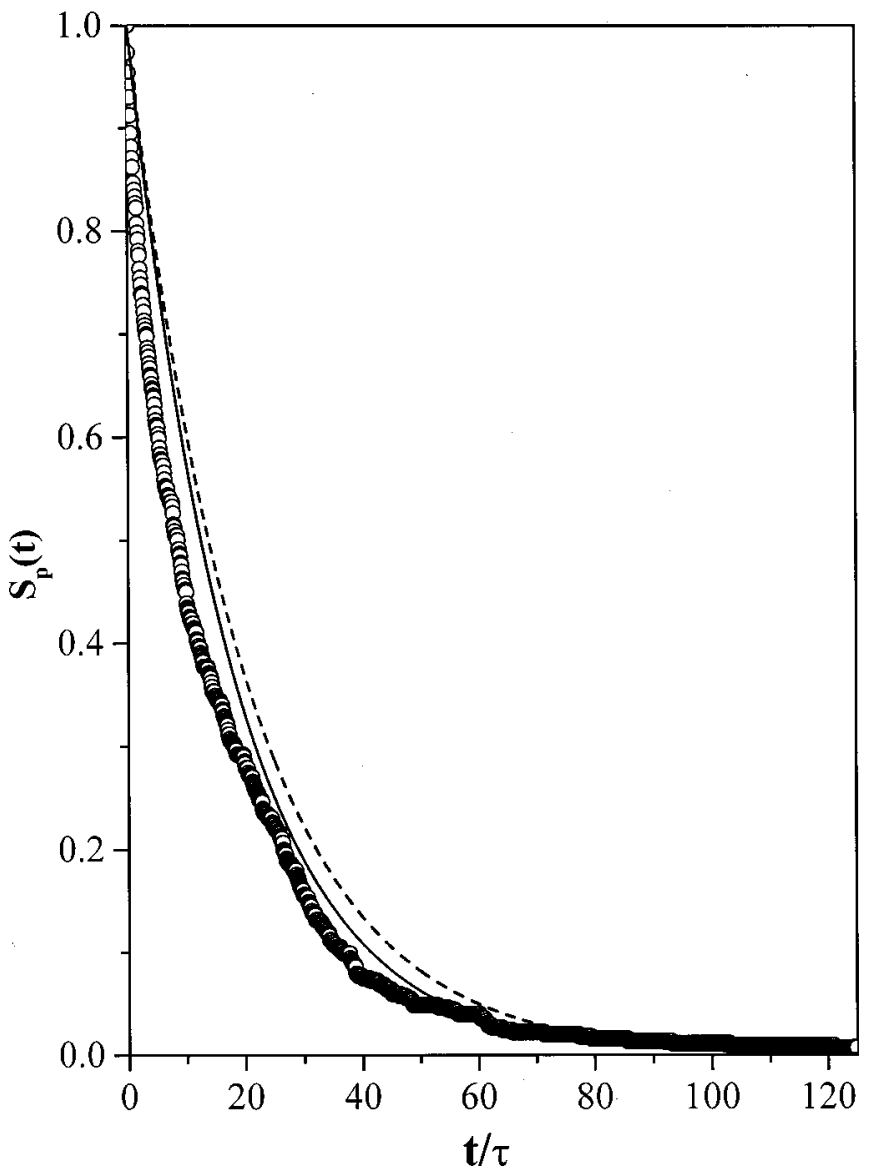

FIG. 4. Comparison of $S_{p}(t)$ at a larger $k_{\text {rad }}$ value namely $k_{\text {rad }}=10$ at $R_{F}$ $=1$ for $N=50$. Symbols represent the simulation result while the full and dashed lines correspond to the prediction of RGF and WF methods, respectively.

remains in few cases, the results obtained from the new scheme are in better agreement with that of the simulations when compared to WF theory results. Note that the discretized sink method gives the same results as that of WF theory in the case of a delta function sink.

The techniques employed in this work could be employed in other related fields. The distance dependent rate appears in several other chemical processes, where the rate of transfer is known to show an exponential distance dependence. One such example is the electron-transfer reactions. It is of interest to use the method employed here to that problem as well. Finally, the simulations results obtained here should be analyzed by using the variational theory of Portman and Wolynes. ${ }^{22}$ Work in these directions is under progress.

\section{ACKNOWLEDGMENTS}

This work is supported in part by the Council of Scientific and Industrial Research (CSIR) and the Department of Science and Technology (DST), India. One of the authors (G.S.) thanks CSIR, New Delhi, India for a research fellowship. 


\section{APPENDIX A: THE REDUCED GREEN'S FUNCTION $\mathrm{G}_{0}\left(\mathrm{R}, t \mid \mathbf{R}_{0}, \mathbf{0}\right)$}

In the Rouse model, one has the modes $X_{p \alpha},(p$ $=1,2, \ldots$ and $\alpha=x, y, z)$ which are the normal modes of the chain. We do not include $p=0$, which is the center-of-mass motion of the chain. These modes have the propagator [see Doi and Edwards, Eqs. (4.18) to (4.22)]

$$
\begin{aligned}
G_{0}\left(X_{p \alpha}, t \mid X_{p \alpha}^{0}, 0\right)= & {\left[\frac{2 \pi k_{B} T}{k_{p}}\left(1-\exp \left(-2 t \lambda_{p}\right)\right)\right]^{-1 / 2} } \\
& \times \exp \left[-\frac{k_{p}\left(X_{p \alpha}-X_{p \alpha}^{0} \exp \left(-t \lambda_{p}\right)\right)^{2}}{2 k_{B} T\left(1-\exp \left(-2 t \lambda_{p}\right)\right)}\right]
\end{aligned}
$$

[see Eq. (3.90) of Doi and Edwards (We deviate slightly from their notation.)].

$$
\lambda_{p}=\frac{3 \pi^{2} k_{B} T}{\zeta N^{2} b^{2}} p^{2}=3 D\left(\frac{\pi}{N b}\right)^{2} p^{2},
$$

and

$$
k_{p}=\frac{6 \pi^{2} k_{B} T}{N b^{2}} p^{2} .
$$

The full propagator is a product over all the $p$ modes. Now $\mathbf{R}$ may be expressed in terms of the vectors $\mathbf{X}_{p}$ as

$$
\mathbf{R}=-4 \sum_{p}^{\prime} \mathbf{X}_{p}
$$

The prime in $\Sigma_{p}^{\prime}$ indicates that we need to sum over only the odd values of $p$. We now evaluate the function $\mathrm{G}_{0}\left(\mathbf{R}, t \mid \mathbf{R}_{0}, 0\right)$. By definition,

$\mathrm{G}_{0}\left(\mathbf{R}, t \mid \mathbf{R}_{0}, 0\right)$

$$
\begin{aligned}
& =\int d \mathbf{S} \int d \mathbf{S}_{0} \mathcal{G}_{0}\left(\mathbf{R}, \mathbf{S}, t \mid \mathbf{R}_{0}, \mathbf{S}_{0}, 0\right) P_{\mathrm{eq}}\left(\mathbf{S}_{0} \mid \mathbf{R}_{0}\right) \\
& =\int d \mathbf{S} \int d \mathbf{S}_{0} \mathcal{G}_{0}\left(\mathbf{R}, S, t \mid \mathbf{R}_{0}, \mathbf{S}_{0}, 0\right) P_{\mathrm{eq}}\left(\mathbf{R}_{0}, \mathbf{S}_{0}\right) / P_{\mathrm{eq}}\left(\mathbf{R}_{0}\right) .
\end{aligned}
$$

Now consider the numerator of the above equation. It is:

$$
I=\int d \mathbf{S} \int d \mathbf{S}_{0} \mathcal{G}_{0}\left(\mathbf{R}, \mathbf{S}, t \mid \mathbf{R}_{0}, \mathbf{S}_{0}, 0\right) P_{\mathrm{eq}}\left(\mathbf{R}_{0}, \mathbf{S}_{0}\right) .
$$

This may be written in terms of the normal modes as

$$
\begin{aligned}
I\left(\mathbf{R}, \mathbf{R}_{0}\right)= & \prod_{a} \prod_{p}^{\prime} \int d X_{p \alpha} \delta\left(R_{\alpha}+4 \sum_{p}^{\prime} X_{p \alpha}\right) \\
& \times \int d X_{p \alpha}^{0} \delta\left(R_{0 \alpha}+4 \sum_{p}^{\prime} X_{p \alpha}^{0}\right) \\
& \times \mathrm{G}_{0}\left(X_{p \alpha}, t \mid X_{p \alpha}^{0}, 0\right) P_{\mathrm{eq}}\left(X_{p \alpha}^{0}\right) .
\end{aligned}
$$

It is easy to evaluate using the integral representation of the Dirac delta function and the $G_{0}\left(X_{p \alpha}, t \mid X_{p \alpha}^{0}, 0\right)$ given previously. Then, one gets

$$
\begin{aligned}
I\left(\mathbf{R}, \mathbf{R}_{0}\right)= & \prod_{\alpha}(1 / 2 \pi)^{2} \int d u_{\alpha} \int d v_{\alpha} \exp \left[i u_{\alpha} R_{\alpha}\right. \\
& \left.+i v_{\alpha} R_{0 \alpha}\right] \prod_{p}^{\prime} \int d X_{p \alpha} \int d X_{p \alpha}^{0} G_{0}\left(X_{p \alpha}, t \mid X_{p \alpha}^{0}, 0\right) \\
& \times G_{0}\left(X_{p \alpha}, \infty \mid 0,0\right) \exp \left[i u_{\alpha} X_{p \alpha}+i v_{\alpha} X_{p \alpha}^{0}\right] .
\end{aligned}
$$

On performing the integration over $X_{p \alpha}$ and $X_{p \alpha}^{0}$, and then over $u_{\alpha}$ and $v_{\alpha}$, we get

$$
I\left(\mathbf{R}, \mathbf{R}_{0}\right)=\frac{e^{-\left(\mathbf{R}^{2}+\mathbf{R}_{0}^{2}-2 \mathbf{R} \cdot \mathbf{R}_{0} \rho(t)\right) / 4 k\left(1-\rho(t)^{2}\right)}}{\left(4 \pi k \sqrt{1-\rho(t)^{2}}\right)^{3}},
$$

where

$$
\begin{aligned}
& k=8 k_{B} T \sum_{p}^{\prime} 1 / k_{p}=N b^{2} / 6=L^{2} / 6, \\
& \rho(t)=\frac{\sum_{p}^{\prime} \exp \left(-\lambda_{p} t\right) / k_{p}}{\sum_{p}^{\prime} 1 / k_{p}}=\frac{8}{\pi^{2}} \sum_{p}^{\prime} \exp \left(-\lambda_{p} t\right) / p^{2} .
\end{aligned}
$$

Now the equilibrium distribution $P_{\text {eq }}\left(\mathbf{R}_{0}\right)$ may be obtained from the above-mentioned, by integrating over $\mathbf{R}$. Thus,

$$
P_{\text {eq }}\left(\mathbf{R}_{0}\right)=\int d \mathbf{R} I\left(\mathbf{R}, \mathbf{R}_{0}\right)=\frac{e^{-\left(\mathbf{R}_{0}^{2}\right) / 4 k}}{(2 \sqrt{\pi k})^{3}} .
$$

Using Eqs. (A2) and (A3) in Eq. (A1) gives

$$
\begin{aligned}
G_{0}\left(\mathbf{R}, t \mid \mathbf{R}_{0}, 0\right)= & \left(\frac{3}{2 \pi L^{2}\left(1-\rho(t)^{2}\right)}\right)^{3 / 2} \\
& \times \exp -\frac{3\left(\mathbf{R}-\mathbf{R}_{0} \rho(t)\right)^{2}}{2 L^{2}\left(1-\rho(t)^{2}\right)},
\end{aligned}
$$

which is the expression used in the paper.

\section{APPENDIX B: LIMITS FOR THE TIME DEPENDENT DIFFUSION, $D(t)$}

By substituting $\rho(t)$ in Eq. (27) we get,

$$
D(t)=\frac{4 D_{0}}{N} \sum_{l ; \text { odd }} \exp \left(-\lambda_{l} t\right) .
$$

From the above-mentioned expression we can obtain both short-time and long-time limits for $D(t)$ as follows. At $t$ $=0$, since the summation in the previous equation can be replaced by $N / 2$, we get

$$
D(t=0)=2 D_{0} .
$$

On the other hand, in $t \rightarrow \infty$ limit, since only the first term in the summation survives, we can write,

$$
\lim _{t \rightarrow \infty} D(t)=\frac{-L^{2}}{3}\left(-\lambda_{1} t\right)
$$

by substituting $\lambda_{1}$ value, we get,

$$
\lim _{t \rightarrow \infty} D(t)=\frac{\pi^{2} D_{0}}{2 N} .
$$


${ }^{1}$ Th. Förster, Ann. Phys. (Leipzig) 2, 55 (1948).

${ }^{2}$ Th. Förster, in Modern Quantum Chemistry, Istanbul Lectures, Part III: Action of Light and Organic Crystals, edited by O. Sinanoglu (Academic, New York, 1965).

${ }^{3}$ R. W. Pastor, R. Zwanzig, and A. Szabo, J. Chem. Phys. 105, 3878 (1996).

${ }^{4}$ G. Wilemski and M. Fixman, J. Chem. Phys. 60, 866 (1974).

${ }^{5}$ G. Wilemski and M. Fixman, J. Chem. Phys. 60, 878 (1974).

${ }^{6}$ M. Doi, Chem. Phys. 11, 107 (1975); S. Sunagawa and M. Doi, Polym. J. (Singapore) 7, 604 (1975); ibid. 8, 239 (1976).

${ }^{7}$ A. Szabo, J. Phys. Chem. 93, 6929 (1989); A. Szabo, K. Schulten, and Z. Schulten, J. Chem. Phys. 72, 4350 (1980).

${ }^{8}$ G. Srinivas, A. Yethiraj, and B. Bagchi, J. Chem. Phys. 114, 9170 (2001).

${ }^{9}$ L. Stryer and R. P. Haugland, Proc. Natl. Acad. Sci. U.S.A. 58, 719 (1967).

${ }^{10}$ C. R. Cantor and P. Pechukas, Proc. Natl. Acad. Sci. U.S.A. 68, 2099 (1971).

${ }^{11}$ A. A. Deniz, T. A. Laurence, G. S. Beligere, M. Dahan, A. B. Martin, D. S. Chemla, P. E. Dawson, P. G. Schultz, and S. Weiss, Proc. Natl. Acad. Sci. U.S.A. 97, 5179 (2000).

${ }^{12}$ M. I. Wallace, L. Ying, S. Balasubramanian, and D. Klenermen, J. Phys. Chem. B 104, 11551 (2000); ibid. 104, 5171 (2000).

${ }^{13}$ D. S. Talaga, W. L. Lau, H. Roder, J. Tang, W. F. DeGrado, and R. M.
Hoschstrasser, Proc. Natl. Acad. Sci. U.S.A. 97, 13021 (1999).

${ }^{14}$ M. A. Winnik, Langmuir 13, 3103 (1997); M. A. Winnik, J. P. S. Farinha, and J. M. G. Martinho, J. Phys. Chem. A 101, 1787 (1997).

${ }^{15}$ R. A. Marcus and N. Sutin, Biochim. Biophys. Acta 811, 275 (1995); R. A. Marcus, Annu. Rev. Phys. Chem. 15, 155 (1964).

${ }^{16}$ M. Bixon, J. Jortner, and J. W. Verhoeven, J. Am. Chem. Soc. 116, 7349 (1994).

${ }^{17} \mathrm{Ph}$. Tamarat, A. Maali, B. Lounis, and M. Orrit, J. Phys. Chem. A 104, 1 (2000).

${ }^{18}$ R. Zwanzig, Acc. Chem. Res. 23, 148 (1990), and references therein.

${ }^{19}$ P. de Gennes, Scaling Concepts in Polymer Physics (Cornell University Press, Ithaca, NY, 1979).

${ }^{20}$ P. E. Rouse, J. Chem. Phys. 21, 1272 (1953).

${ }^{21}$ M. Doi and S. F. Edwards, The Theory of Polymer Dynamics (Oxford University Press, Oxford, 1986).

${ }^{22}$ J. J. Portman and P. G. Wolynes, J. Phys. Chem. A 103, 10602 (1999); J. Wang and P. G. Wolynes, Phys. Rev. Lett. 74, 4317 (1994).

${ }^{23}$ D. J. Bicout and A. Szabo, J. Chem. Phys. 1209, 2325 (1998)

${ }^{24}$ R. A. Denny and B. Bagchi, J. Phys. Chem. A 103, 9061 (1999); R. A. Denny, B. Bagchi, and P. F. Barbara, J. Chem. Phys. 115, 6058 (2001).

${ }^{25}$ A. Samantha and S. K. Gosh, Phys. Rev. E 47, 4568 (1993).

${ }^{26}$ B. Bagchi and N. Gayathri, Adv. Chem. Phys. 107, 1 (2000). 